Article

\title{
A Facile Synthesis of Fully Protected meso-Diaminopimelic Acid (DAP) and Its Application to the Preparation of Lipophilic $N$-Acyl iE-DAP
}

\author{
Yukako Saito, Yuichi Yoshimura, Hideaki Wakamatsu and Hiroki Takahata * \\ Laboratory of Organic and Pharmaceutical Chemistry, Faculty of Pharmaceutical Sciences, \\ Tohoku Pharmaceutical University, 4-4-1 Komatsushima, Aoba-ku, Sendai 981-8558, Japan; \\ E-Mails: yukako-s@tohoku-pharm.ac.jp (Y.S.); yoshimura@tohoku-pharm.ac.jp (Y.Y.); \\ hiwaka@tohoku-pharm.ac.jp (H.W.) \\ * Author to whom correspondence should be addressed; E-Mail: takahata@tohoku-pharm.ac.jp; \\ Tel./Fax: +81-22-727-0144.
}

Received: 5 December 2012; in revised form: 8 January 2013 / Accepted: 9 January 2013 / Published: 16 January 2013

\begin{abstract}
Synthesis of beneficial protected meso-DAP 9 by cross metathesis of the Garner aldehyde-derived vinyl glycine $\mathbf{1 b}$ with protected allyl glycine $\mathbf{2}$ in the presence of Grubbs second-generation catalyst was performed. Preparation of lipophilic $N$-acyl iE-DAP as potent agonists of NOD 1-mediated immune response from 9 is described.
\end{abstract}

Keywords: protected meso-diaminopimelic acid; $\mathrm{N}$-acyl iE-DAP; cross metathesis

\section{Introduction}

Peptidoglycan (PGN) is an essential component of the cell walls of virtually all bacteria. The function of the PGN is to preserve cell integrity by withstanding the internal osmotic pressure [1,2]. The biosynthesis of PGN is a well-recognized target for antibiotic development [3]. Bacterial cell wall PGN can function as a potent immunostimulator and an adjuvant for antibody production. PGN partial structures are recognized by the intracellular nucleotide-binding oligomerization domain proteins 1 and 2 (NOD1 and NOD2) that mediate host recognition of bacterial molecules [4-6]. The recognition core of Nod1 stimulatory molecules is $\gamma$-D-glutamyl-meso-diaminopimelic acid (iE-DAP) which is a constituent of most Gram-negative and some Gram-positive bacteria. In addition, synthetic, lipophilic, $\mathrm{N}$-acyl iE-DAP derivatives have been shown to be potent NOD 1 agonists [7]. Thus, DAP scaffold 
peptides would be expected to function as NOD 1 agonists. In connection with our interest in the synthesis of DAP [8], we report herein on a new synthesis of orthogonally protected meso-DAP and applications to preparing $N$-acyl iE-DAP from protected iE-DAP (Figure 1).

Figure 1. Structures of meso-DAP and iE-DAP.
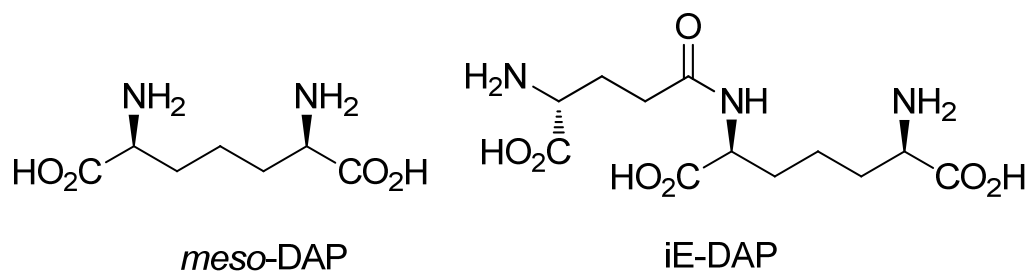

\section{Results and Discussion}

Because DAP-containing peptides such as iE-DAP and FK565 [9,10] have significant biological activities and functions, the synthesis of orthogonally protected meso-DAP as synthetic intermediates has been a subject of considerable interest by several groups [11-26]. However, available methods suffer from several disadvantages, including the use of commercially inaccessible starting materials, harsh reaction conditions, multi steps, and lower product yields. We envisaged that orthogonally protected meso-DAP could be conveniently prepared by a cross metathesis (CM) between the readily available Garner aldehyde-derived vinyl glycine equivalent $\mathbf{1}$ and protected allyl glycine $\mathbf{2}$ (Scheme 1).

Scheme 1. Cross metathesis of Garner aldehyde-derived vinyl glycine equivalents $\mathbf{1}$ and protected allyl glycine 2 .<smiles>[R]C=C[C@H]1COC(C)(C)[NH2+][C@H]1C</smiles>

$1 a: R=H$

$1 \mathrm{~b}: \mathrm{R}=\mathrm{Me}$

1c: $\mathrm{R}=\mathrm{CO}_{2} \mathrm{Et}$

1d: $\mathrm{R}=\mathrm{CH}_{2} \mathrm{OH}$

1e $: \mathrm{R}=\mathrm{Ph}$

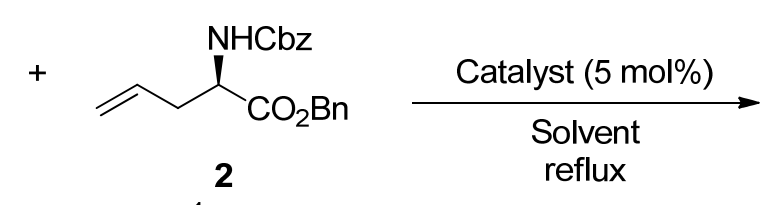

1 eq.

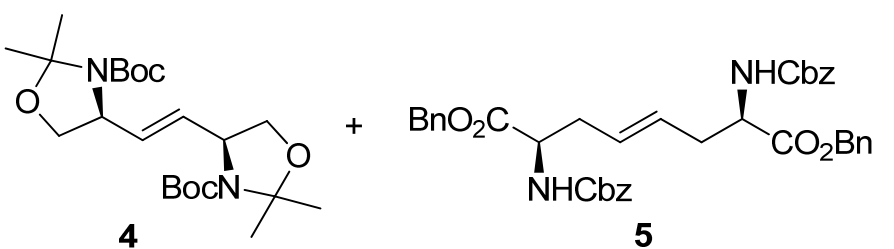

A variety of substituted olefins 1a-e as vinyl glycine equivalents were obtained from the Garner aldehyde in high yields according to the corresponding literature reports [27-31]. It is expected that a homocoupling of 1 would scarcely occur due to a bulkiness of $N$-Boc-oxazolidine. First, a coupling began with the CM of 1a with 2 [32,33]. Treatment of 1 a $(5 \mathrm{mmol})$ with $\mathbf{2}(1 \mathrm{mmol})$ using the Grubbs second-generation catalyst $\mathbf{A}\left(5 \mathrm{~mol} \%\right.$, Figure 2) in $\mathrm{CH}_{2} \mathrm{Cl}_{2}$ under reflux gave a desired product 3 in $56 \%$ together with homo-coupling products traces of 4 and $\mathbf{5}(22 \%)$ (entry 1 in Table 1$)$. 
Figure 2. Catalysts for cross metathesis.

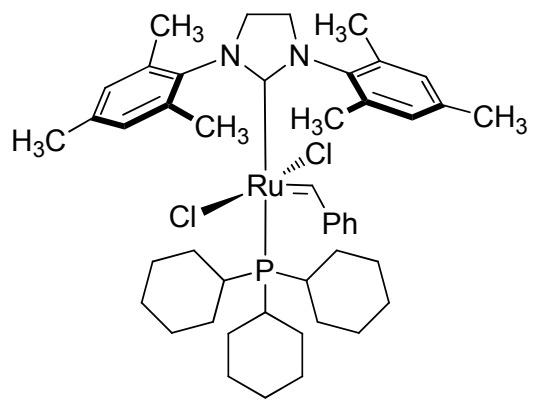

A : Grubbs 2nd generation

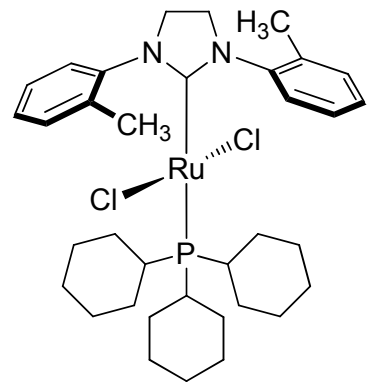

B

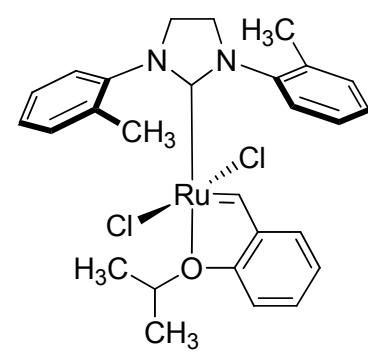

C

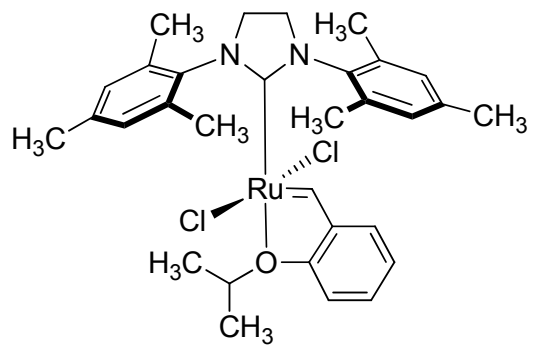

D : Hoveyda-Grubbs 2nd generation

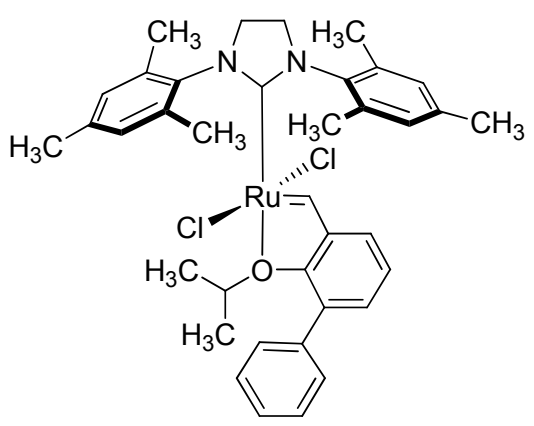

E : Blechert' catalyst

Table 1. CM of 1a with 2.

\begin{tabular}{cccccc}
\hline Entry & $\mathbf{1}$ & Catalyst & Solvent & Time (h) & Yield (\%) \\
\hline 1 & $\mathbf{1 a}$ & $\mathrm{A}$ & $\mathrm{CH}_{2} \mathrm{Cl}_{2}$ & 7 & 56 \\
2 & $\mathbf{1 a}$ & $\mathrm{B}$ & $\mathrm{CH}_{2} \mathrm{Cl}_{2}$ & 4.5 & 28 \\
3 & $\mathbf{1 a}$ & $\mathrm{C}$ & $\mathrm{CH}_{2} \mathrm{Cl}_{2}$ & 36 & 33 \\
4 & $\mathbf{1 a}$ & $\mathrm{A}$ & Toluene & 3 & 64 \\
\hline
\end{tabular}

All reactions were carried out with a ratio $(\mathbf{1 a : 2}=5: 1)$ under reflux.

Next, the use of a combination of catalysts B [34] and C [34] gave lower yields (28\% and 33\%) of 3, respectively (entries 2 and 3). On the other hand, when toluene was used as the solvent in place of $\mathrm{CH}_{2} \mathrm{Cl}_{2}$, a higher yield (64\%) of 3 was obtained (entry 4).

In addition, the use of CM using several substituted olefins $\mathbf{1 b}-\mathbf{e}$ as vinyl glycine units in toluene was examined and the results are shown in Table 2 . The use of $\mathbf{1 b}(E: Z=1: 10)$ gave the best yield (76\%) of 3. Unfortunately, CM using other derivatives, such as $\mathbf{1 c}-\mathbf{e}$, resulted in lower yields (entries 4 6). Furthermore, the CM of $\mathbf{1 b}$ with the Hoveyda-Grubbs 2nd generation D [34] and the Blechert $\mathbf{E}$ [35] catalysts afforded lower yields (56\% and 9\%), respectively. Accordingly CM in conjunction with a combination of $\mathbf{1 b}$ and $\mathbf{A}$ as a catalyst resulted in better yields. Additionally, CM using the pure $E$ isomer and the $Z$ isomer of $\mathbf{1 b}$ resulted in nearly the same yields (75\%) (entry 7). Furthermore, the $\mathrm{CM}$ in Table 2 produced no the homocoupling product 4 as expected.

With 3 in hand, our interest was focused on the synthesis of orthogonally protected meso-DAP. The hydrogenation of 3 in the presence of $\mathrm{PtO}_{2}$ as a catalyst gave 6, which was transformed by hydrolysis of the amino acetal with with $p$ - $\mathrm{TsOH}$ in aqueous $\mathrm{MeOH}$ into the alcohol 7 in $81 \%$ yield in two steps. 
The primary alcohol of 7 was converted into the carboxylic acid 8 by oxidation with TEMPO, which was esterfied, without isolation, with benzyl alcohol using our developed 1-tert-butoxy-2-tertbutoxycarbonyl-1,2-dihydroisoquinoline (BBDI) [36] to yield the fully protected meso-DAP 9 in $94 \%$ yield in two-steps.

Table 2. CM of Garner aldehyde-derived vinyl glycine equivalents $\mathbf{1 b}-\mathbf{e}$ with $\mathbf{2}$.

\begin{tabular}{ccccc}
\hline Entry & 1 & Catalyst & Time (h) & Yield (\%) \\
\hline 1 & 1b & A & 4 & 76 \\
2 & $\mathbf{1 b}$ & $\mathbf{D}$ & 3 & 56 \\
3 & $\mathbf{1 b}$ & $\mathbf{E}$ & 4 & 9 \\
4 & $\mathbf{1 c}$ & $\mathbf{A}$ & 4 & trace \\
5 & $\mathbf{1 d}$ & $\mathbf{A}$ & 3 & 9 \\
6 & $\mathbf{1 e}$ & $\mathbf{A}$ & 3 & 10 \\
7 & $\mathbf{1 b}(\boldsymbol{E}: \boldsymbol{Z}=\mathbf{1 0 : 1})$ & 4 & 75 \\
\hline
\end{tabular}

All reactions were carried out with a ratio $(\mathbf{1}: 2=5: 1)$ under reflux in toluene.

Having the desired 9 in hand, we embarked on the synthesis of $N$-acyl iE-DAP, which is known to function as a strong agonist for the stimulation of NOD 1 [6]. Deprotection of the tert-butoxycarbonyl group of 9 by treatment with trifluoroacetic acid followed by condensation of the resulting amine 10 [37] with Fmoc-D-Glu-OBn [38,39] using EDC in the presence of HOBT and triethylamine gave the protected iE-DAP 11 in 54\% yield in two steps. Next, 11 was treated with diethylamine to afford the deprotected amine 12, which was subsequently acylated with capryloyl chloride and myristoyl chloride to produce the corresponding $N$-acyl derivatives $\mathbf{1 3}$ and $\mathbf{1 4}$ in $68 \%$ and $89 \%$ yields, respectively. Finally, the deprotection of $\mathbf{1 3}$ and $\mathbf{1 4}$ with $\mathrm{Pd}(\mathrm{OH})_{2}$ as the catalyst under hydrogen gave $N$-capryloyl iE-DAP 15 and $N$-myristoyl iE-DAP 16, respectively, in quantitative yields (Scheme 2).

Scheme 2. Synthesis of $N$-acyl iE-DAP.
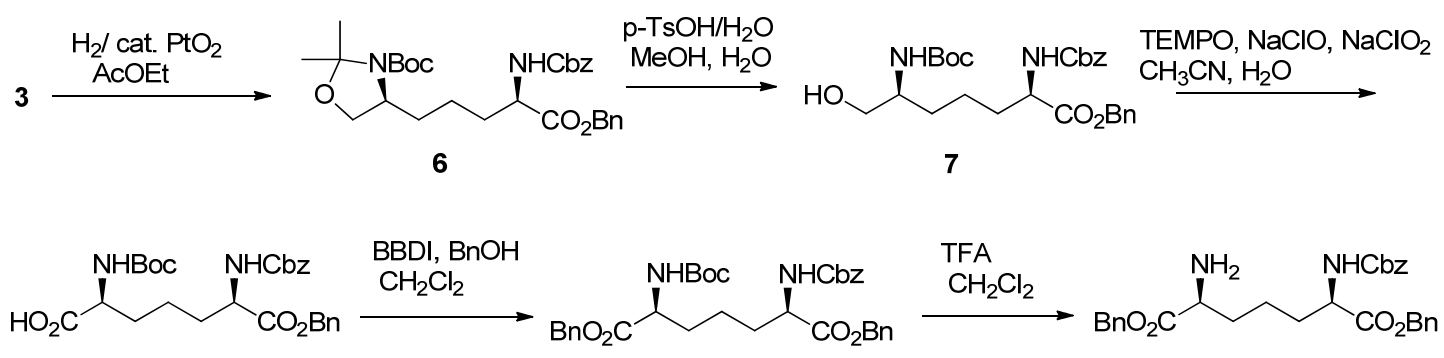

8

9

10

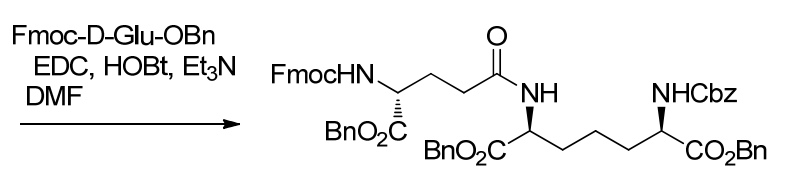

11

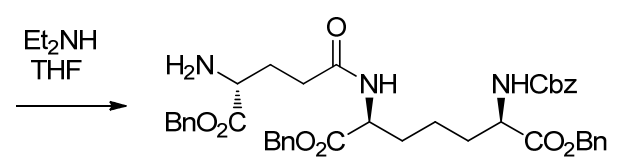

12

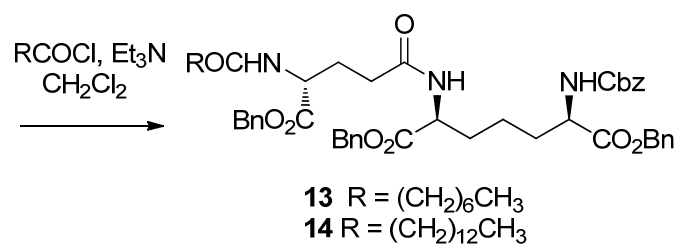

$14 \mathrm{R}=\left(\mathrm{CH}_{2}\right)_{12} \mathrm{CH}_{3}$

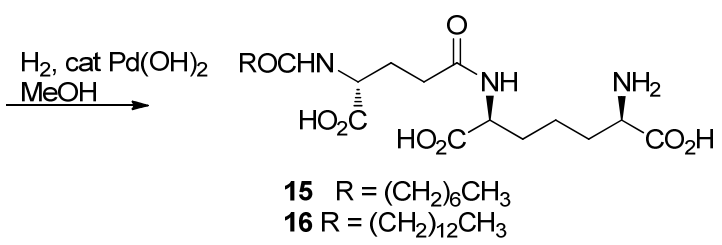




\section{Experimental}

\subsection{General}

Infrared (IR) spectra were recorded on a Perkin-Elmer 1600 series FT-IR spectrometer. Mass spectra (MS) were recorded on a JEOL JMN-DX 303/JMA-DA 5000 spectrometer (Tokyo, Japan). Microanalyses were performed on a Perkin-Elmer CHN 2400 Elemental Analyzer (Tokyo, Japan). Optical rotations were measured with a JASCO DIP-360 or JASCO P-1020 digital polarimeter. Proton nuclear magnetic resonance ( $\left.{ }^{1} \mathrm{H}-\mathrm{NMR}\right)$ spectra were recorded on JEOL JNM-EX 270 (270 MHz) or JEOL JNM-AL 400 (400 MHz) or JNM-LA (600 Mhz) spectrometer (Tokyo, Japan), using tetramethylsilane as an internal standard. The following abbreviations are used: $\mathrm{s}=$ singlet, $\mathrm{d}=$ doublet, $\mathrm{t}=$ triplet, $\mathrm{q}=$ quartet, $\mathrm{m}=$ multiplet, $\mathrm{br}=$ broad. Column chromatography was carried out on Merck Silica gel 60 (230-400 mesh) or KANTO Silica Gel 60N (40-50 $\mu \mathrm{m})$ for flash chromatography.

\subsection{Synthesis}

(S)-tert-butyl 2,2-dimethyl-4-vinyloxazolidine-3-carboxylate (1a) [27], (S)-tert-butyl 2,2-dimethyl-4(prop-1-en-1-yl)oxazolidine-3-carboxylate (1b) [28], (S)-tert-butyl 4-(3-ethoxy-3-oxoprop-1-en-1-yl)2,2-dimethyloxazolidine-3-carboxylate (1c) [29], (S)-tert-butyl 4-(3-hydroxyprop-1-en-1-yl)-2,2dimethyloxazolidine-3-carboxylate (1d) [30], (S)-tert-butyl 2,2-dimethyl-4-styryloxazolidine-3carboxylate (1e) [31] and (S)-benzyl 2-(((benzyloxy)carbonyl)amino)pent-4-enoate (2) [32,33] were prepared by the reported procedures.

(S)-tert-Butyl 4-((R)-5-(benzyloxy)-4-(benzyloxycarbonylamino)-5-oxopent-1-enyl)-2,2-dimethyloxazolidine-3-carboxylate (3), (4S,4'S)-di-tert-butyl 4,4'-((E)-ethene-1,2-diyl)bis(2,2dimethyloxazolidine-3-carboxylate) (4), and (2R,7R,E)-dibenzyl 2,7bis(((benzyloxy)carbonyl)amino)oct-4-enedioate (5)

To a solution of $N$-Cbz- $(R)$-allylglycine benzyl ester (124 mg, $0.36 \mathrm{mmol})$ and $(Z)-(S)-N$-Boc-2,2dimethyl-4-(1-propenyl)oxazolidine (408 mg, $1.8 \mathrm{mmol})$ in dry $\mathrm{CH}_{2} \mathrm{Cl}_{2}(1.8 \mathrm{~mL})$ was added tricyclohexylphosphine[1,3-bis(2,4,6-trimethylphenyl)-4,5-dihydroimidazol-2-yl-idene][benzylidine]ruthenium (IV) dichloride (2nd Grubbs catalyst) $(15 \mathrm{mg}, 0.018 \mathrm{mmol})$. After the mixture was refluxed for $7 \mathrm{~h}$, the solvent was evaporated. The residue was chromatographed using $(n$-hexane/AcOEt $=2: 1)$ as eluent to yield 4 (17 mg), 3 (109 mg, 56\%), and 5 (26 mg, 22\%).

3: $[\alpha]^{25}+9.73^{\circ}\left(c\right.$ 1.49, $\left.\mathrm{CHCl}_{3}\right)$. IR (neat) $\mathrm{cm}^{-1}: 3329,2937,1726,1697,1389,1366,1255,1176$. ${ }^{1} \mathrm{H}-\mathrm{NMR}\left(400 \mathrm{MHz}, \mathrm{CDCl}_{3}\right) \delta 1.31-1.56(15 \mathrm{H}, \mathrm{m}), 2.53-2.60(2 \mathrm{H}, \mathrm{m}), 3.62(1 \mathrm{H}, \mathrm{dd}, J=2.17,8.93 \mathrm{~Hz})$, $3.97(1 \mathrm{H}, \mathrm{dd}, J=6.04,8.94 \mathrm{~Hz}), 4.17(0.5 \mathrm{H}, \mathrm{br} \mathrm{s}), 4.30(0.5 \mathrm{H}$, br s$), 4.44-4.49(1 \mathrm{H}, \mathrm{m}), 5.06-5.24$ $(4 \mathrm{H}, \mathrm{m}), 5.35-5.56(3 \mathrm{H}, \mathrm{m}), 7.34(10 \mathrm{H}, \mathrm{s}) .{ }^{13} \mathrm{C}-\mathrm{NMR}\left(100 \mathrm{MHz}, \mathrm{CDCl}_{3}\right) \delta$ 23.4, 24.8, 26.5, 27.2, 28.1, 28.3, 34.6, 58.6, 66.7, 66.8, 67.0, 67.8, 67.9, 68.1, 79.5, 80.1, 93.4, 93.8, 125.1, 125.6, 128.0, 128.1, 128.1, 128.4, 128.5, 134.1, 135.1, 136.0, 151.7, 155.6, 171.3. EI-MS $m / z 538\left(\mathrm{M}^{+}\right)$. Anal. Calcd for $\mathrm{C}_{30} \mathrm{H}_{38} \mathrm{~N}_{2} \mathrm{O}_{7}$ : C, 66.90; H, 7.11; N, 5.20. Found : C, 66.80; H, 7.31; N, 5.38. 
4: $[\alpha]^{23}{ }_{\mathrm{D}}+31.5^{\circ}\left(c 0.9, \mathrm{CHCl}_{3}\right) .{ }^{1} \mathrm{H}-\mathrm{NMR}\left(400 \mathrm{MHz}, \mathrm{CDCl}_{3}\right) 1.43-1.83(30 \mathrm{H}, \mathrm{m}), 3.37(2 \mathrm{H}, \mathrm{d}, \mathrm{J}=8.7 \mathrm{~Hz})$, 4.03-4.05 (2H, m), 4.29-4.44 (2H, m), $5.59\left(1 \mathrm{H}\right.$, br s). EI-MS $m / z 426\left(\mathrm{M}^{+}\right)$. HRMS Calcd for $\mathrm{C}_{22} \mathrm{H}_{38} \mathrm{~N}_{2} \mathrm{O}_{6}: 426.2730$. Found 426.2728 .

5: m.p. $73-74{ }^{\circ} \mathrm{C} .[\alpha]^{24}{ }_{\mathrm{D}}-4.31^{\circ}\left(c 1.05, \mathrm{CHCl}_{3}\right)$. IR $(\mathrm{KBr}) \mathrm{cm}^{-1}: 3320,3064,3035,2956,1741,1692$, 1536, 1346, 1262, 1221, 1052. ${ }^{1} \mathrm{H}-\mathrm{NMR}\left(400 \mathrm{MHz}, \mathrm{CDCl}_{3}\right) \delta$ 2.35-2.46 (4H, m), 4.40-4.45 (2H, m), 5.08-5.18 (8H, m), 5.24-5.26 (2H, m), $5.40(2 \mathrm{H}$, br d, $J=7.73 \mathrm{~Hz}), 7.32(20 \mathrm{H}, \mathrm{s}) .{ }^{13} \mathrm{C}-\mathrm{NMR}(100 \mathrm{MHz}$, $\left.\mathrm{CDCl}_{3}\right)$ 35.3, 53.4, 66.9, 67.2, 128.1, 128.1, 128.4, 128.5, 128.5 128.6, 135.2, 136.2, 155.7, 171.4. EI-MS $m / z 650\left(\mathrm{M}^{+}\right)$. HRMS Calcd for $\mathrm{C}_{38} \mathrm{H}_{38} \mathrm{~N}_{2} \mathrm{O}_{8}$ : 650.2628. Found 650.2624.

To a solution of $N$-Cbz- $(R)$-allylglycine benzyl ester $(139 \mathrm{mg}, 0.41 \mathrm{mmol})$ and $(Z)-(S)-N$-Boc-2,2dimethyl-4-(1-propenyl)oxazolidine (497 mg, $2.06 \mathrm{mmol}$ ) in dry toluene $(3 \mathrm{~mL}$ ) was added 2nd Grubbs catalyst (18 mg, $0.021 \mathrm{mmol})$. After the mixture was refluxed for $4 \mathrm{~h}$, the solvent was evaporated. The residue was chromatographed using ( $n$-hexane/AcOEt $=3: 1)$ as eluent to yield $3(168 \mathrm{mg}, 76 \%)$.

(2R,6S)-Benzyl 2-(benzyloxycarbonylamino)-6-(tert-butoxycarbonylamino)-7-hydroxyheptanoate (6)

A mixture of $3(263 \mathrm{mg}, 0.49 \mathrm{mmol})$ in the presence of $\mathrm{PtO}_{2}(1.8 \mathrm{mg}, 0.02 \mathrm{mmol})$ under hydrogen atomosphere in AcOEt $(3.5 \mathrm{~mL})$ was stirred for $14 \mathrm{~h}$ at room temperature. After the mixture was filtered through Celite, the filtrate was evaporated to provide $(S)$-tert-Butyl 4-((R)-5-(benzyloxy)-4(benzyloxycarbonylamino)-5-oxopentyl)-2,2-dimethyloxazolidine-3-carboxylate (6) (257 mg, 97\%) as an oil. $[\alpha]^{25}+11.18^{\circ}\left(c 1.2, \mathrm{CHCl}_{3}\right)$. IR (neat) $\mathrm{cm}^{-1}: 3340,2979,2938,1695,1532,1456,1392,1366$, 1257, 1210, 1174. ${ }^{1} \mathrm{H}-\mathrm{NMR}\left(400 \mathrm{MHz}, \mathrm{CDCl}_{3}\right) \delta 1.26-1.84(21 \mathrm{H}, \mathrm{m}), 3.61-3.67(1.5 \mathrm{H}, \mathrm{m}), 3.82(1 \mathrm{H}$, br s), $4.41(1 \mathrm{H}$, br s), $5.09(2 \mathrm{H}, \mathrm{s}), 5.15(2 \mathrm{H}, \mathrm{s}), 5.42(0.5 \mathrm{H}$, br d, $J=7.25 \mathrm{~Hz}), 5.60(0.5 \mathrm{H}$, br d, $J=6.76 \mathrm{~Hz}), 7.33(10 \mathrm{H}, \mathrm{s}) .{ }^{13} \mathrm{C}-\mathrm{NMR}\left(100 \mathrm{MHz}, \mathrm{CDCl}_{3}\right) \delta 21.8,23.1,24.4,26.7,27.5,28.3,29.6$, $32.0,32.4,32.5,33.1,53.8,56.8,57.0,66.7,66.8,66.9,67.1,79.4,80.1,93.1,93.6,128.0,128.2$, $128.4,128.5,135.2,139.2,152.3,155.9,172.1$. EI-MS $m / z 540\left(\mathrm{M}^{+}\right)$.

Seven drops of water were added to a mixture of carboxylic acid (299 $\mathrm{mg}, 0.55 \mathrm{mmol}$ ) and $p$ - $\mathrm{TsOH} \cdot \mathrm{H}_{2} \mathrm{O}(0.07 \mathrm{mmol}, 13 \mathrm{mg})$ in $\mathrm{MeOH}(5 \mathrm{~mL})$ and then the mixture was stirred for $36 \mathrm{~h}$ at room temperature. The whole was evaporated. AcOEt $(40 \mathrm{~mL})$ was added to the residue. The mixture was successively washed with sat. $\mathrm{NaHCO}_{3}(10 \mathrm{~mL})$ and brine $(10 \mathrm{~mL})$, dried over $\mathrm{Na}_{2} \mathrm{SO}_{4}$, and evaporated. The residue was purified with chromatography using ( $n$-hexane/AcOEt $=1: 1)$ as eluent to yield 6 (225 mg, 81\%) as solid. M.p. $66-67{ }^{\circ} \mathrm{C}$. $[\alpha]^{25} \mathrm{D}-2.27^{\circ}\left(c 1.0, \mathrm{CHCl}_{3}\right)$. IR ( $\left.\mathrm{KBr}\right) \mathrm{cm}^{-1}: 3353$, 2939, 1742, 1694, 1537, 1289, 1247, 1171, 1048. ${ }^{1} \mathrm{H}-\mathrm{NMR}\left(400 \mathrm{MHz}, \mathrm{CDCl}_{3}\right) \delta 1.31-1.42(13 \mathrm{H}, \mathrm{m})$, 1.54-1.70 (1H, m), 1.80-1.89 (1H, m), 3.45-3.55 (3H, m), 4.39-4.44 (1H, m), 4.83 (1H, br s), 5.09 $(2 \mathrm{H}, \mathrm{s}), 5.12-5.21(2 \mathrm{H}, \mathrm{m}), 5.57(1 \mathrm{H}, \mathrm{br} \mathrm{s}), 7.30-7.36(10 \mathrm{H}, \mathrm{m}) .{ }^{13} \mathrm{C}-\mathrm{NMR}\left(100 \mathrm{MHz}, \mathrm{CDCl}_{3}\right) \delta 21.3$, 28.3, 30.5, 32.5, 52.0, 53.3, 64.5, 67.0, 67.1, 79.4, 128.0, 128.1, 128.2, 128.4, 128.4, 128.6, 135.2, 136.1, 156.2, 156.2, 172.2. EI-MS $m / z 500\left(\mathrm{M}^{+}\right)$. HRMS Calcd for $\mathrm{C}_{27} \mathrm{H}_{36} \mathrm{~N}_{2} \mathrm{O}_{7}$ : 500.2523. Found 500.2503 . 
(2R,6S)-Dibenzyl 2-(benzyloxycarbonylamino)-6-(tert-butoxycarbonylamino)heptanedioate (9)

2,2,6,6-Tetramethylpiperidine 1-oxyl (TEMPO) $(7 \mathrm{mg}, 0.045 \mathrm{mmol})$ and $2 \mathrm{M} \mathrm{NaClO}_{2}(0.43 \mathrm{~mL}$, $0.86 \mathrm{mmol})$ were added to a solution of $7(225 \mathrm{mg}, 0.45 \mathrm{mmol})$ in $\mathrm{MeCN}$ : sodium phosphate buffer consisted of a 1:1 mixture of $0.67 \mathrm{M} \mathrm{NaH}_{2} \mathrm{PO}_{4}$ and $0.67 \mathrm{M} \mathrm{Na}_{2} \mathrm{HPO}_{4}(\mathrm{pH}=6.5)(3: 2,6.5 \mathrm{~mL})$ at $40{ }^{\circ} \mathrm{C}$. A diluted solution of commercial bleach $(25.5 \mu \mathrm{L})$ in $\mathrm{H}_{2} \mathrm{O}(485 \mu \mathrm{L})$ was then added to the gradually over $0.5 \mathrm{~h}$, and the reaction was stirred at $40{ }^{\circ} \mathrm{C}$ for $18 \mathrm{~h}$. The reaction was cooled to room temperature and quenched with sat. $a q . \mathrm{Na}_{2} \mathrm{SO}_{3}$ until the mixture became colorless. The solvent was evaporated, the aqueous mixture acidified to $\mathrm{pH}<3$ with $1 \mathrm{M} \mathrm{HCl}$ and extracted with ether $(10 \mathrm{~mL})$ five times. Organic layers were washed with brine, dried overNa $\mathrm{SO}_{4}$ and evaporated to yield (2S,6R)-7(benzyloxy)-6-(((benzyloxy)carbonyl)amino)-2-((tert-butoxycarbonyl)amino)-7-oxoheptanoic acid (8). Without further purification, a mixture of 8 and BBDI (164 mg, $0.54 \mathrm{mmol}$ ) and benzyl alcohol (48 $\mathrm{mg}$, $0.45 \mathrm{mmol})$ in $\mathrm{CH}_{2} \mathrm{Cl}_{2}(2 \mathrm{~mL})$ was stirred for $19 \mathrm{~h}$ at room temperature. AcOEt (40 mL) was added to the mixture and the whole was successively washed with $5 \% \mathrm{HCl}(10 \mathrm{~mL})$ and brine $(10 \mathrm{~mL})$. The solvent was dried over $\mathrm{Na}_{2} \mathrm{SO}_{4}$ and evaporated. The residue was purified with chromatography using $n$-hexane/AcOEt (3:1) to yield 7 (256 mg, 94\%, 2 steps). M.p. $82-83{ }^{\circ} \mathrm{C}$. $[\alpha]^{24}{ }_{\mathrm{D}}-0.28^{\circ}\left(\mathrm{c} 1.4, \mathrm{CHCl}_{3}\right)$. IR (neat) $\mathrm{cm}^{-1}: 3345,2955,1741,1716,1525,1367,1215,1167 .{ }^{1} \mathrm{H}-\mathrm{NMR}\left(400 \mathrm{MHz}, \mathrm{CDCl}_{3}\right) \delta$ 1.22-1.42 (11H, m), 1.53-1.67 (2H, m), 1.75-1.88 (2H, m), 4.26-4.31 (1H, m), 4.34-4.39 (1H, m), $5.02(1 \mathrm{H}$, br d, $J=7.25 \mathrm{~Hz}), 5.06-5.20(6 \mathrm{H}, \mathrm{m}), 5.33(1 \mathrm{H}, \mathrm{br} \mathrm{d}, J=7.25 \mathrm{~Hz}), 7.26-7.34(15 \mathrm{H}, \mathrm{m})$. ${ }^{13} \mathrm{C}-\mathrm{NMR}\left(100 \mathrm{MHz}, \mathrm{CDCl}_{3}\right) \delta$ 20.8, 28.2, 31.8, 32.1, 53.0, 53.6, 67.0, 67.1, 79.9, 128.1, 128.1, 128.3, 128.4, 128.5, 128.6, 128.6, 135.2, 135.3, 136.1, 155.4, 155.9, 172.0, 172.3. EI-MS $m / z 604\left(\mathrm{M}^{+}\right)$. Anal. Calcd for $\mathrm{C}_{34} \mathrm{H}_{40} \mathrm{~N}_{2} \mathrm{O}_{8}: \mathrm{C}, 67.53 ; \mathrm{H}, 6.67 ; \mathrm{N}, 4.63$. Found: $\mathrm{C}, 67.29 ; \mathrm{H}, 6.81 ; \mathrm{N}, 4.43$.

\section{(2S,6R)-Dibenzyl 2-((R)-4-(((9H-fluoren-9-yl)methoxy)carbonylamino)-5-(benzyloxy)-5-oxopentan-} amido)-6-(benzyloxycarbonylamino)heptanedioate (11)

TFA $(0.6 \mathrm{~mL})$ was added to a mixture of $9(78 \mathrm{mg}, 0.13 \mathrm{mmol})$ in $\mathrm{CH}_{2} \mathrm{Cl}_{2}(0.7 \mathrm{~mL})$ and then the mixture was stirred for $2 \mathrm{~h}$ at room temperature. $\mathrm{CH}_{2} \mathrm{Cl}_{2}(5 \mathrm{~mL})$ was added to the reaction mixture and the whole neutralized with sat. $\mathrm{NaHCO}_{3}$. The mixture was extracted with $\mathrm{CH}_{2} \mathrm{Cl}_{2}$, dried over $\mathrm{Na}_{2} \mathrm{SO}_{4}$ and evaporated to yield an amine 10. Fmoc-D-Glu benzyl ester (59 mg, $0.129 \mathrm{mmol}$ ) was added to a solution of the prepared amine 10 in DMF $(1.5 \mathrm{~mL}) . \mathrm{Et}_{3} \mathrm{~N}(20 \mu \mathrm{L}, 0.142 \mathrm{mmol})$, HOBt $(21 \mathrm{mg}$, $0.155 \mathrm{mmol})$, and $\mathrm{EDC} \cdot \mathrm{HCl}(27 \mathrm{mg}, 0.142 \mathrm{mmol})$ were successively added to the mixture at $-20{ }^{\circ} \mathrm{C}$. The reaction was stirred for $20 \mathrm{~min}$ at the same temperature and then was done at room temperature for $20 \mathrm{~h}$. After addition of AcOEt $(100 \mathrm{~mL})$, the mixture was successively washed with $1 \mathrm{~N} \mathrm{HCl}$, brine $(10 \mathrm{~mL} \times 2)$, water $(10 \mathrm{~mL})$, and $5 \% \mathrm{NaHCO}_{3}(10 \mathrm{~mL})$. The organic layer was dried over $\mathrm{Na}_{2} \mathrm{SO}_{4}$ and evaporated. The residue was purified with chromatography using ( $n$-hexane/AcOEt $=1: 1)$ as eluent to yield $11\left(70 \mathrm{mg}, 57 \%, 2\right.$ steps) as an oil. $[\alpha]^{23}{ }_{\mathrm{D}}-2.0^{\circ}\left(c 1.6, \mathrm{CHCl}_{3}\right)$. IR (neat) $\mathrm{cm}^{-1}: 3367,2920,2851$, 1734, 1722, 1662, 1534, 1250, 1211. ${ }^{1} \mathrm{H}-\mathrm{NMR}\left(400 \mathrm{MHz}, \mathrm{CDCl}_{3}\right) \delta 1.30-1.39(1 \mathrm{H}, \mathrm{m}), 1.56-1.67$ $(3 \mathrm{H}, \mathrm{m}), 1.77-1.84(2 \mathrm{H}, \mathrm{m}), 1.91-2.00(1 \mathrm{H}, \mathrm{m}), 2.20(3 \mathrm{H}$, br s), 4.14-4.19 $(1 \mathrm{H}, \mathrm{m}), 4.32-4.48(3 \mathrm{H}$, m), $4.55(1 \mathrm{H}, \mathrm{dd}, J=7.24,13.04 \mathrm{~Hz}), 5.06-5.18(9 \mathrm{H}, \mathrm{m}), 5.34(1 \mathrm{H}$, br d, $J=7.24 \mathrm{~Hz}), 5.71(1 \mathrm{H}$, br d, $J=7.73 \mathrm{~Hz}), 6.51(1 \mathrm{H}$, br d, $J=6.76 \mathrm{~Hz}), 7.29-7.40(24 \mathrm{H}, \mathrm{m}), 7.58(2 \mathrm{H}$, br d, $J=7.25 \mathrm{~Hz}), 7.75(2 \mathrm{H}$, br d, $J=7.73 \mathrm{~Hz}) .{ }^{13} \mathrm{C}-\mathrm{NMR}\left(100 \mathrm{MHz}, \mathrm{CDCl}_{3}\right) \delta 20.8,28.6,31.3,31.9,32.0,47.1,51.9,53.3,53.5$, 
$66.9,67.0,67.1,119.9,119.9,125.1,127.0,127.7,128.0,128.1,128.2,128.2,128.4,128.5,128.6$, 135.1, 135.2, 135.2, 136.1, 141.2, 141.2, 143.6, 143.8, 156.0, 156.3, 171.8, 171.8, 171.9, 171.9. FAB-MS $m / z 946\left(\mathrm{M}^{+}+1\right)$. HRMS Calcd for $\mathrm{C}_{56} \mathrm{H}_{55} \mathrm{~N}_{3} \mathrm{O}_{11}$ : 946.3870. Found 946.3934.

(2S,6R)-Dibenzyl 2-((R)-4-heptanecarbonylamino-5-(benzyloxy)-5-oxopentanamido)-6(benzyloxycarbonylamino)heptanedioate (13)

$\mathrm{Et}_{2} \mathrm{NH}(2.5 \mathrm{~mL})$ was gradually added to a solution of 11 (62 $\left.\mathrm{mg}, 0.066 \mathrm{mmol}\right)$ in THF (1 mL) with ice cooling over $20 \mathrm{~min}$. The reaction was stirred at room temperature for $3 \mathrm{~h}$ and then was evaporated. The residue was purified with chromatography using $\left(\mathrm{CHCl}_{3} / \mathrm{MeOH}=100: 1\right)$ as eluent to yield $(2 S, 6 R)$ Dibenzyl 2-((R)-4-amino-5-(benzyloxy)-5-oxopentanamido)-6-(benzyloxycarbonylamino)heptanedioate (12). $[\alpha]^{24}{ }_{\mathrm{D}}-3.1^{\circ}\left(c\right.$ 1.0, $\left.\mathrm{CHCl}_{3}\right)$. IR (neat) $\mathrm{cm}^{-1}: 3309,2925,1739,1648,1534,1215 .{ }^{1} \mathrm{H}-\mathrm{NMR}$ (400 MHz, $\left.\mathrm{CDCl}_{3}\right) \delta 1.21-1.41(2 \mathrm{H}, \mathrm{m}), 1.56-1.66(3 \mathrm{H}, \mathrm{m}), 1.74-1.83(3 \mathrm{H}, \mathrm{m}), 2.06-2.11(1 \mathrm{H}, \mathrm{m}), 2.21-2.28$ (1H, m), 2.32-2.38 (1H, m), 3.45-3.49 (1H, m), 4.32-4.37 (1H, m), 4.55-4.60 (1H, m), 5.07-5.15 $(8 \mathrm{H}, \mathrm{m}), 5.38(1 \mathrm{H}$, br d, $J=7.73 \mathrm{~Hz}), 6.59(1 \mathrm{H}, \mathrm{br} \mathrm{d}, J=7.73 \mathrm{~Hz}), 7.30-7.33(20 \mathrm{H}, \mathrm{m}) .{ }^{13} \mathrm{C}-\mathrm{NMR}$ $\left(100 \mathrm{MHz}, \mathrm{CDCl}_{3}\right) \delta 20.8,29.7,31.6,31.9,32.4,51.7,53.5,53.5,66.7,67.0,67.1,67.1,128.0,128.1$, 128.2, 128.3, 128.3, 128.4, 128.4, 128.5, 128.6, 128.6, 135.2, 135.5, 136.1, 156.0, 172.0, 172.0, 172.1, 175.4. FAB-MS $m / z 724\left(\mathrm{M}^{+}+1\right)$. HRMS Calcd for $\mathrm{C}_{41} \mathrm{H}_{45} \mathrm{~N}_{3} \mathrm{O}_{9}: 724.3189$. Found 724.3230.

$\mathrm{Et}_{3} \mathrm{~N}(9 \mu \mathrm{L}, 0.063 \mathrm{mmol})$ and $n$-octanoyl chloride $(9 \mu \mathrm{L}, 0.053 \mathrm{mmol})$ were added to a solution of the amine 12 in $\mathrm{CH}_{2} \mathrm{Cl}_{2}(1 \mathrm{~mL})$ with ice cooling. The reaction was stirred at room temperature for $7 \mathrm{~h}$ and then evaporated. After addition of AcOEt $(30 \mathrm{~mL})$, the mixture was washed with $10 \%$ citric acid $(10 \mathrm{~mL})$ and brine $(10 \mathrm{~mL})$. The organic solvent was dried over $\mathrm{Na}_{2} \mathrm{SO}_{4}$ and evaporated. The residue was purified with chromatography using ( $n$-hexane/AcOEt $=1: 1)$ ) as eluent to yield $\mathbf{1 3}(38 \mathrm{mg}, 68 \%$, 2 steps) as an oil. ${ }^{1} \mathrm{H}-\mathrm{NMR}\left(400 \mathrm{MHz}, \mathrm{CDCl}_{3}\right) \delta 0.87(3 \mathrm{H}, \mathrm{t}, J=6.76 \mathrm{~Hz}), 1.19-1.41(8 \mathrm{H}, \mathrm{m}), 1.54-1.71$ $(6 \mathrm{H}, \mathrm{m}), 1.75-1.93(2 \mathrm{H}, \mathrm{m}), 2.16-2.24(6 \mathrm{H}, \mathrm{m}), 4.33-4.38(1 \mathrm{H}, \mathrm{m}), 4.55(1 \mathrm{H}, \mathrm{dd}, J=7.25,12.57 \mathrm{~Hz})$, 4.73-4.77 (1H, m), 5.09-5.15 (8H, m), $5.50(1 \mathrm{H}$, br d, $J=8.21 \mathrm{~Hz}), 6.46(1 \mathrm{H}$, br d, $J=7.25 \mathrm{~Hz}), 7.04$ $(1 \mathrm{H}$, br d, $J=7.73 \mathrm{~Hz}), 7.29-7.33(20 \mathrm{H}, \mathrm{m})$. FAB-MS $m / z 850\left(\mathrm{M}^{+}+1\right)$. HRMS Calcd for $\mathrm{C}_{49} \mathrm{H}_{59} \mathrm{~N}_{3} \mathrm{O}_{10}: 850.4234$. Found 850.4278 .

(2S,6R)-Dibenzyl 2-((R)-4-tridecanecarbonylamino-5-(benzyloxy)-5-oxopentanamido)-6(benzyloxycarbonylamino)heptanedioate (14)

According to the procedure described for a preparation of 13, a treatment of 11 (71 $\mathrm{mg}, 0.075 \mathrm{mmol})$ with $\mathrm{Et}_{2} \mathrm{NH}(2 \mathrm{~mL})$ gave 12 (39 mg, 72\%), which was converted with $\mathrm{Et}_{3} \mathrm{~N}(8 \mu \mathrm{L}, 0.058 \mathrm{mmol})$ and myristoyl chloride $(16 \mu \mathrm{L}, 0.058 \mathrm{mmol})$ into $14(40 \mathrm{mg}, 89 \%)$ as a solid. M.p. $90-92{ }^{\circ} \mathrm{C} .[\alpha]^{27}{ }_{\mathrm{D}}-4.6^{\circ}$ (c 1.3, $\left.\mathrm{CHCl}_{3}\right) .{ }^{1} \mathrm{H}-\mathrm{NMR}\left(400 \mathrm{MHz}, \mathrm{CDCl}_{3}\right) \delta{ }^{1} \mathrm{H}-\mathrm{NMR}\left(400 \mathrm{MHz}, \mathrm{CDCl}_{3}\right) \delta 0.88(3 \mathrm{H}, \mathrm{t}, J=6.76 \mathrm{~Hz})$, 1.24-1.41 (22H, m), 1.56-1.92 (8H, m), 2.16-2.23 (4H, m), 4.33-4.36 (1H, m), 4.53-4.58 (1H, m), 4.71-4.77 (1H, m), 5.07-5.18 (8H, m), $5.55(1 \mathrm{H}$, br d, $J=8.21 \mathrm{~Hz}), 6.51(1 \mathrm{H}$, br d, $J=7.73 \mathrm{~Hz}), 7.06$ $(1 \mathrm{H}$, br d, $J=7.73 \mathrm{~Hz}), 7.29-7.32(20 \mathrm{H}, \mathrm{m}) .{ }^{13} \mathrm{C}-\mathrm{NMR}\left(100 \mathrm{MHz}, \mathrm{CDCl}_{3}\right) \delta 14.1,21.0,22.6,25.6$, 29.0, 29.2, 29.3, 29.3, 29.5, 29.6, 29.6, 29.6, 31.1, 31.8, 31.9, 32.2, 36.5, 51.6, 52.0, 53.6, 67.0, 67.0, 67.1, 67.3, 128.0, 128.1, 128.2, 128.4, 128.4, 128.5, 128.6, 128.6, 135.1, 135.2, 135.3, 136.2, 156.0, 172.0, 172.1, 173.9. FAB-MS $m / z 935\left(\mathrm{M}^{+}+2\right)$. Anal. Calcd for $\mathrm{C}_{55} \mathrm{H}_{71} \mathrm{~N}_{3} \mathrm{O}_{10}: \mathrm{C}, 70.71 ; \mathrm{H}, 7.66 ; \mathrm{N}$, 4.50. Found C, 70.73; H, 7.90; N, 4.48. 
(2R,6S)-2-Amino-6-((R)-4-carboxy-4-octanamidobutanamido)heptanedioic acid (15)

A suspension of $13(34 \mathrm{mg}, 0.04 \mathrm{mmol})$ in the presence $\mathrm{Pd}(\mathrm{OH})_{2}(25 \mathrm{mg})$ under hydrogen atomosphere in $\mathrm{MeOH}(1.5 \mathrm{~mL})$ was stirred for $3 \mathrm{~h}$. The mixture was filtered through Celite. The filtrate was evaporate to yield 15 quantitatively as an oil. $[\alpha]^{23}{ }_{\mathrm{D}}-1.3^{\circ}\left(c 1.0, \mathrm{MeOH}: \mathrm{CHCl}_{3}=1: 1\right)$. ${ }^{1} \mathrm{H}-\mathrm{NMR}\left(400 \mathrm{MHz}, \mathrm{CDCl}_{3}\right) \delta 0.90(3 \mathrm{H}, \mathrm{t}, J=6.28 \mathrm{~Hz}), 1.31-1.32(10 \mathrm{H}, \mathrm{m}), 1.52-1.64(4 \mathrm{H}, \mathrm{m})$, 1.73-1.76 (1H, m), 1.85-2.04 (4H, m), 2.14-2.19 (1H, m), 2.23-2.27 (2H, m), 2.35-2.38 (2H, m), 3.68 (br s, 1H), 4.37-4.40 (2H, m). ${ }^{13} \mathrm{C}-\mathrm{NMR}\left(100 \mathrm{MHz}, \mathrm{CDCl}_{3}\right) \delta 14.4,22.6,23.7,26.9,28.7,30.2,30.3$, $31.6,32.3,32.9,33.1,36.9,53.5,53.6,55.4,173.8,175.0,175.4,175.6,176.4$. FAB-MS $m / z 446$ $\left(\mathrm{M}^{+}+1\right)$.

(2R,6S)-2-Amino-6-((R)-4-carboxy-4-tetradecanamidobutanamido)heptanedioic acid (16)

A suspension of $14(32 \mathrm{mg}, 0.034 \mathrm{mmol})$ in the presence $\mathrm{Pd}(\mathrm{OH})_{2}(20 \mathrm{mg})$ under hydrogen atomosphere in $\mathrm{MeOH}(1.5 \mathrm{~mL})$ was stirred for $3 \mathrm{~h}$. The mixture was filtered through Celite. The filtrate was evaporate to yield $\mathbf{1 6}$ quantitatively as an oil. IR (neat) $\mathrm{cm}^{-1}: 3425,2919,2850,1710$, 1637, 1538. ${ }^{1} \mathrm{H}-\mathrm{NMR}\left(400 \mathrm{MHz}, \mathrm{CD}_{3} \mathrm{OD}\right) \delta 0.89(3 \mathrm{H}, \mathrm{t}, J=6.76 \mathrm{~Hz}), 1.28-1.31(20 \mathrm{H}, \mathrm{m}), 1.52-1.63$ $(4 \mathrm{H}, \mathrm{m}), 1.69-1.79(1 \mathrm{H}, \mathrm{m}), 1.83-2.02(4 \mathrm{H}, \mathrm{m}), 2.14-2.18(1 \mathrm{H}, \mathrm{m}), 2.24(2 \mathrm{H}, \mathrm{t}, J=7.25 \mathrm{~Hz}), 2.36$ $(2 \mathrm{H}, \mathrm{t}, J=7.49 \mathrm{~Hz}), 3.78-3.81(1 \mathrm{H}, \mathrm{m}), 4.36-4.44(2 \mathrm{H}, \mathrm{m}) .{ }^{13} \mathrm{C}-\mathrm{NMR}\left(100 \mathrm{MHz}, \mathrm{CD}_{3} \mathrm{OD}\right) \delta 14.4$, 22.6, 23.7, 26.9, 28.6, 30.3, 30.5, 30.5, 30.7, 30.8, 30.8, 30.8, 31.3, 32.2, 33.1, 33.1, 36.8, 53.3, 53.4, 54.7, 172.9, 175.0, 175.1, 175.3, 176.5. FAB-MS m/z $530\left(\mathrm{M}^{+}+1\right)$. HRMS Calcd for $\mathrm{C}_{26} \mathrm{H}_{47} \mathrm{~N}_{3} \mathrm{O}_{8}$ : 530.3447. Found 530.3397.

\section{Conclusions}

In summary, a concise synthesis of $N$-acyl iE-DAP was accomplished starting from the protected meso-DAP 9 as a convenient synthon via the use of CM between the Garner aldehyde-derived vinyl glycine $\mathbf{1 b}$ and the protected allyl glycine $\mathbf{2}$ in nine steps in yields in the range of $22 \% \sim 29 \%$.

\section{Acknowledgments}

This work was supported in part by a grant of Strategic Research Foundation Grant-aided Project for Private Universities from Ministry of Education, Culture, Sport, Science, and Technology, Japan (MEXT).

\section{References}

1. Rietschel, E.T.; Schletter, J.; Weidemann, B.; El-Samalouti, V.; Mattern, T.; Zahringer, U.; Seydel, U.; Brade, H.; Flad, H.D.; Kusumoto, S.; et al. Lipopolysaccharide and peptidoglycan: CD14-dependent bacterial inducers of inflammation. J. Microb. Drug Resist. 1998, 4, 37-44.

2. Van Heijenoort, J. Formation of the glycan chains in the synthesis of bacterial peptidoglycan. Glycobiology 2001, 11, 25R-36R.

3. Bugg, T.D.H.; Braddick, D.; Dowson, C.G.; Roper, D.I. Bacterial cell wall assembly: Still an attractive antibacterial target. Trends Biotechnol. 2011, 29, 167-173. 
4. Chamaillard, M.; Hashimoto, M.; Horie, Y.; Masumoto, J.; Qiu, S.; Saab, L.; Ogura, Y.; Kawasaki, A.; Fukase, K.; Kusumoto, S. An essential role for NOD1 in host recognition of bacterial peptidoglycan containing diaminopimelic acid. Nat. Immunol. 2003, 4, 702-707.

5. Girardin, S.E.; Travassos, L.H.; Herve, M.; Blanot, D.; Boneca, I.G.; Philpott, D.J.; Sansonetti, P.J.; Mengin-Lecreulx, D. Peptidoglycan molecular requirements allowing detection by Nod1 and Nod 2. J. Biol. Chem. 2003, 278, 41702-41708.

6. Masumoto, J.; Yang, K.; Varambally, S.; Hasegawa, M.; Tomlins, S.A.; Qiu, S.; Fujimoto, Y.; Kawasaki, A.; Foster, S.J.; Horie, Y.; et al. Nod1 acts as an intracellular receptor to stimulate chemokine production and neutrophil recruitment in vivo. J. Exp. Med. 2006, 203, 203-213.

7. Hasegawa, M.; Kawasaki, A.; Yang, K.; Fujimoto, Y.; Masumoto, J.; Breukink, E.; Nunez, G.; Fukase, K.; Inohara, N. A Role of Lipophilic Peptidoglycan-related Molecules in Induction of Nod1-mediated Immune Responses. J. Biol. Chem. 2007, 282, 11757-11764.

8. Saito, Y.; Shinkai, T.; Yoshimura, Y.; Takahata, H. A straightforward stereoselective synthesis of meso-, $(S, S)$ - and $(R, R)$-2,6-diaminopimelic acids from cis-1,4-diacetoxycyclohept-2-ene. Bioorg. Med. Chem. Lett. 2007, 17, 5894-5896.

9. Gotoh, T.; Nakahara, K.; Iwami, M.; Aoki, H.; Imanaka, H. Studies on a new immunoactive peptide, FK-156. I. Taxonomy of the producing strains. J. Antibiot. 1982, 35, 1280-1285.

10. Gotoh, T.; Nakahara, K.; Nishiura, T.; Hashimoto, M.; Kino, T.; Kuroda, Y.; Okuhara, M.; Kohsaka, M.; Aoki, H.; Imanaka, H. Studies on a new immunoactive I peptide, FK-156 II. Fermentation, extraction and chemical and biological characterization. J. Antibiot. 1982, 35, 1286-1292.

11. Jurgens, A.R. Asymmetric synthesis of differentially protected meso-2,6-diaminopimelic acid. Tetrahedron Lett. 1992, 33, 4727-4730.

12. Williams, R.M.; Yuan, C. Asymmetric synthesis of 2,6-diaminopimelic acids. J. Org. Chem. 1992, 57, 6519-6527.

13. Holcomb, R.C.; Schow, S.; Ayral-Kaloustian, S.; Powell, D. An asymmetric synthesis of differentially protected meso-2,6-diaminopimelic acid. Tetrahedron Lett. 1994, 35, 7005-7008.

14. Gao, Y.; Lane-Bell, P.; Vederas, J.C. Stereoselective Synthesis of meso-2,6-Diaminopimelic Acid and Its Selectively Protected Derivatives. J. Org. Chem. 1998, 63, 2133-2143.

15. Davis, F.A.; Srirajan, V. Asymmetric Synthesis of $(2 S, 6 S)$ - and meso-(2S,6R)-Diaminopimelic Acids from Enantiopure Bis(sulfinimines). J. Org. Chem. 2000, 65, 3248-3251.

16. Paradisi, F.; Porzi, G.; Rinaldi, S.; Sandri, S. A simple asymmetric synthesis of (+)- and (-)-2,6diaminopimelic acids. Tetrahedron: Asymmetry 2000, 11, 1259-1262.

17. Collier, P.N.; Patel, I.; Taylor, R.J.K. A concise, stereoselective synthesis of meso-2,6diaminopimelic acid (DAP). Tetrahedron Lett. 2001, 42, 5953-5954.

18. Collier, P.N.; Campbell, A.D.; Patel, I.; Raynham, T.M.; Taylor, R.J.K. Enantiomerically Pure $\alpha$-Amino Acid Synthesis via Hydroboration-Suzuki Cross-Coupling. J. Org. Chem. 2002, 67, 1802-1815.

19. Roberts, J.L.; Chan, C. Asymmetric synthesis of differentially protected meso-2,6-diaminopimelic acid. Tetrahedron Lett. 2002, 43, 7679-7682. 
20. Sutherland, A.; Vederas, J.C. Conjugate addition of radicals generated from diacyloxyiodobenzenesto dehydroamino acid derivatives; a synthesis of diaminopimelic acid analogues. Chem. Commun. 2002, 224-225.

21. Galeazzi, R.; Garavelli, M.; Grandi, A.; Monari, M.; Porzi, G.; Sandri, S. Unusual peptides containing the 2,6-diaminopimelic acid framework: Stereocontrolled synthesis, X-ray analysis, and computational modelling. Part 2. Tetrahedron: Asymmetry 2003, 14, 2639-2649.

22. Spantulescu, M.D.; Jain, R.P.; Derksen, D.J.; Vederas, J.C. Photolysis of Diacyl Peroxides: A Radical-Based Approach for the Synthesis of Functionalized Amino Acids. Org. Lett. 2003, 5, 2963-2965.

23. Del, J.R.; Goodman, M. An Efficient RCM-Based Synthesis of Orthogonally Protected meso-DAP and FK565. J. Org. Chem. 2004, 69, 8946-8948.

24. Chowdhury, A.R.; Boons, G. The synthesis of diaminopimelic acid containing peptidoglycan fragments using metathesis cross coupling. Tetrahedron Lett. 2005, 46, 1675-1678.

25. Kawasaki, A.; Karasudani, Y.; Otsuka, Y.; Hasegawa, M.; Inohara, N.; Fujimoto, Y.; Fukase, K. Synthesis of Diaminopimelic Acid Containing Peptidoglycan Fragments and Tracheal Cytotoxin (TCT) and Investigation of Their Biological Functions. Chem. Eur. J. 2008, 14, 10318-10330.

26. Wang, W.; Xiong, C.; Yang, J.; Hruby, V.J. An efficient synthesis of (2S,6S)- and mesodiaminopimelic acids via asymmetric hydrogenation. Synthesis 2002, 94-98.

27. Banba, Y.; Abe, C.; Nemoto, H.; Kato, A.; Adachi, I.; Takahata, H. Asymmetric synthesis of fagomine and its congeners. Tetrahedron: Asymmetry 2001, 12, 817-819.

28. Collado, I.; Pedregal, C.; Mazon, A.; Espinosa, J.F.; Blanco-Urgoiti, J.; Schoepp, D.D.; Wright, R.A.; Johnson, B.G.; Kingston, A.E. (2S,1'S,2'S,3'R)-2-(2'-Carboxy-3'-methylcyclopropyl) Glycine Is a Potent and Selective Metabotropic Group 2 Receptor Agonist with Anxiolytic Properties. J. Med. Chem. 2002, 45, 3619-3629.

29. Fa Liu, F.; Hu, T.-S.; Yao, Z.-J. Stereoselective synthesis of new modified conformationally constrained L-tyrosine analogue with potential applications to SH2 domain ligands. Tetrahedron 2005, 61, 4971-4981.

30. Devel, L.; Hamon, L.; Becker, H.; Thellend, A.; Vidal-Crosa, A. Synthesis of protected 2-amino2-deoxy-D-xylothionolactam derivatives and some aspects of their reactivity. Carbohydr. Res. 2003, 338, 1591-1601.

31. Schmidtmann, F.W.; Benedum, T.E.; McGarvey, G.J. First Total Synthesis of 1-O- $\beta$-DGlucopyranosyl-5-deoxyadenophorine and Its Aglycon Congener: Determination of the Absolute Configuration. J. Org. Chem. 2004, 69, 1497-1503.

32. Robl, J.A. Heterocyclo-fused [1,3]oxazepines and -thiazepines and analogs as inhibitors of neutral endopeptiadase and angiotensin converting enzyme. U.S. Patent US 5508272 A, 16 April 1996.

33. Robl, J.A.; Kronenthal, D.R.; Goderey, J.D., Jr. Bicyclic carboxylic acids as inhibitors of neutral endopeptidase and angiotensin-converting enzyme. Eur. Patent EP 629627A2, 21 December 1994.

34. Schrodi, Y.; Pederson, R.L. Evolution and Applications of Second-Generation Ruthenium Olefin Metathesis Catalysts. Aldrichimica Acta 2007, 40, 45-52.

35. Wakamatsu, H.; Blechert, S. A Highly Active and Air-Stable Ruthenium Complex for Olefin Metathesis. Angew. Chem. Int. Ed. 2002, 41, 794-796. 
36. Saito, Y.; Watanabe, T.; Takahata, H. A novel 1-tert-butoxy-2-tert-butoxycarbonyl-1,2dihydroisoquinoline (BBDI)-catalyzed esterification of $N$-protected amino acids with nearly equimolar amounts of alcohols in the presence of $\mathrm{Boc}_{2} \mathrm{O}$. Tetrahedron Lett. 2006, 47, 3099-3102.

37. Conversion of $\mathbf{1 0}$ to meso-DAP has been accomplished by hydrogenlysis and the spectral data of hydrochloride salt of prepared meso-DAP was accordance with those of our reported values (ref. [8]).

38. Demaria, S.; Metafora, V.; Metafora, S.; Ravagnan, G.; Carteni, M.; Pontoni, G.; Facchiano, A.;

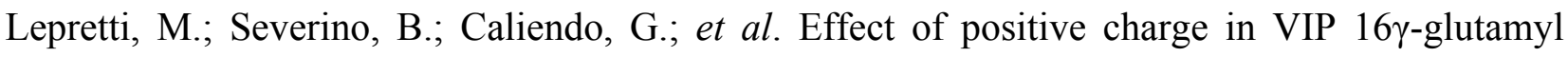
diamino derivatives on hVPAC1 and hVPAC2 receptor function. J. Pept. Sci. 2008, 14, 102-109.

39. Hard, I.; Torres, J.L.; Valencia, G.; Garcia-Anton, J.M.; Reig, F. Synthesis and biological activity of substance P analogues. Int. J. Peptide Protein Res. 1989, 33, 335-339.

Sample Availability: Contact the authors.

(C) 2013 by the authors; licensee MDPI, Basel, Switzerland. This article is an open access article distributed under the terms and conditions of the Creative Commons Attribution license (http://creativecommons.org/licenses/by/3.0/). 